British Journal of Industrial Medicine 1985;42:73-74

\title{
Editorial
}

\section{Vinyl chloride: the evidence for human carcinogenicity in different target organs}

Ever since the original observation of the carcinogenicity of vinyl chloride in man ${ }^{1}$ and animals, ${ }^{23}$ this compound has been studied to determine the nature and mechanism of its effects. The observation of angiosarcoma of the liver in an occupationally exposed group is now universally accepted, and vinyl chloride is included among the relatively small group of compounds whose carcinogenicity is not only deduced on the basis of animal studies but demonstrated in the human population. It should be noted that the workers among whom these cases arose were probably very heavily exposed even by the less stringent standards of a few years ago, and the total number of cases observed remains relatively small in relation to the large number who must have received some exposure to this widely used industrial intermediate. The success of the epidemiological method in demonstrating the problem, and indeed the reason for the initial discovery, depends on the fact that angiosarcoma is an exceedingly rare tumour in the general population. In the absence of any evidence for "thresholds" in the dose response for complete carcinogens, as opposed to promoters, ${ }^{4}$ the risk assessment on which current occupational health standards are based must depend on the anticipated frequency of tumours known to be caused by exposure to this compound.

It is therefore of great interest and importance to consider whether other less specific tumours are also caused by exposure to vinyl chloride, as proposed in a recent publication in this journal. ${ }^{5}$ The rate of incidence of other tumours could be much higher than the rate of angiosarcoma formation, while remaining difficult to demonstrate by epidemiological methods. This is due to the higher background incidence of these other tumours, and the substantial variation in these rates caused by extraneous factors. Various studies have suggested an increase in lung cancer in exposed populations, ${ }^{6}$ but this remains an extremely difficult case to demonstrate owing to the high incidence of lung cancer in the general population and its relation to smoking habits. Even in the study presently discussed, complete smoking histories were not available, and although the authors dismiss this confounding variable as an explanation of their findings others may choose not to do so. On the other hand, the finding of an excess of malignant melanoma in the study population is certainly both interesting and unprecedented. It is also much more difficult to dismiss as the result of confounding factors, owing to the lower background incidence and the fact that the confounding variables (race, sunlight exposure) are connected more with geography than with personal habits and therefore less likely to vary between control and exposed populations. On the other hand, some unexpected associations between this tumour and occupational circumstances, such as the use of fluorescent lighting, have been suggested, if not proved. ${ }^{7}$ The reported association between exposure to vinyl chloride and cancer of the colon and thyroid gland is also of interest in considering the overall risk from this material.

The interpretation of these findings must be tentative in view of the small number of cases and (except in the case of the rarer thyroid tumours) modest ratio of observed to expected cases. It is, none the less, important, since an increase in the incidence of relatively common tumours such as those in the lung and colon could far outnumber the small number of angiosarcomas (one in this study). Animal carcinogenicity studies which have been performed ${ }^{3}$ only partly support the proposition that vinyl chloride is a cause of tumours other than liver angiosarcoma in man. In rats angiosarcoma is observed in the liver and at other sites. Tumours of the brain and kidney are also observed, but there is no obvious animal counterpart to the proposed increase in colonic tumours or melanoma. Although some studies have suggested an increase in skin tumours (other than Zymbal gland tumours), this is far less convincing than the situation in the liver. The lung tumours observed in the original studies in the rat by Viola et $a^{2}{ }^{2}$ were considered by Maltoni and Lefemine ${ }^{3}$ to be metastases from Zymbal gland tumours rather than primary lung neoplasms. Conversely, there is no human anatomical counterpart to the Zymbal gland carcinoma observed in the rat. 
Apart from the Zymbal gland carcinoma and angiosarcomas, the frequency of appearance of tumours in rats exposed by inhalation became significant only at high doses.

In the epidemiological work under consideration it was observed that, at the period during which excess non-angiosarcoma tumours were found in the groups exposed to vinyl chloride, there was also a process on site using the more potent mutagen $^{8}$ and carcinogen 1,2-dichloroethane (ethylene dichloride, EDC). This compound has been shown to cause lung, gastrointestinal, mammary, and subcutaneous tumours in rat and mice at moderate doses in an NCI bioassay. ${ }^{9}$ It should be noted, however, that this was a gavage study rather than one using exposure by inhalation, so the details of organ specificity may not be comparable.

In view of these observations it may be desirable to review the data on which the Norwegian study of polyvinyl chloride workers was based, to determine whether some or all of the tumours other than angiosarcomas of the liver can be explained as a result of exposure to 1,2-dichloroethane. Since there is documentation of other exposure conditions it seems reasonable to hope that this would be possible. The answer to this question has considerable bearing on the presumed increment of risk due to exposure to vinyl chloride at the higher levels documented in this study. The risk implied at the levels of exposure required under current legislation is necessarily lower, but still worthy of consideration.

A G SALMON

TUC Centenary Institute of Occupational Health, London School of Hygiene and Tropical Medicine, Keppel Street, London WC1E 7HT, UK.

\section{References}

' Creech JL, Johnson MN. Angiosarcoma of liver in the manufacture of polyvinyl chloride. J Occup Med 1974;16:150-1.

2 Viola PL, Bigotti A, Caputo A. Oncogenic response of rat skin, lungs and bones to vinyl chloride. Cancer Res 1971;31:516-9.

${ }^{3}$ Maltoni C, Lefemine G. Carcinogenicity bioassays of vinyl chloride: current results. Ann NY Acad Sci 1975;246:195218.

4 Tennekes HA, Edler L, Kunz HW. Dose-response analysis of the enhancement of liver tumour formation in CF-1 mice by dieldrin. Carcinogenesis 1982;3:941-5.

s Storetvedt Heldaas S, Langard SL, Andersen A. Incidence of cancer among vinyl chloride and polyvinyl chloride workers. Br J Ind Med 1984;41:25-30.

- Waxweiler RJ, Stringer W, Wagoner JK, Jones J, Falk H, Carter C. Neoplastic risk among workers exposed to vinyl chloride. Ann NY Acad Sci 1976;271:40-8.

' Beral V, Evans S, Shaw H, Milton G. Malignant melanoma and exposure to fluorescent lighting at work. Lancet 1982;ii:2903.

${ }^{8}$ Rannug U, Ramel C. Mutagenicity of waste products from vinyl chloride industries. Toxicol Environ Health 1977;2:1019-29.

- US National Cancer Institute (Department of Health, Education and Welfare: National Institutes of Health). Bioassay of 1,2dichloroethane for possible carcinogenicity. Washington: DHEW, 1978. (DHEW publication No (NIH) 78-1361, 1978.) 